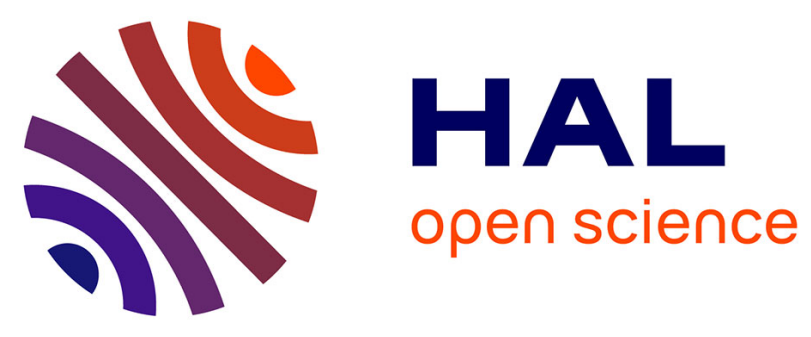

\title{
An Improved Elman Neural Network for Daily Living Activities Recognition
}

Zaineb Liouane, Tayeb Lemlouma, Philippe Roose, Frédéric Weis, Messaoud Hassani

\section{- To cite this version:}

Zaineb Liouane, Tayeb Lemlouma, Philippe Roose, Frédéric Weis, Messaoud Hassani. An Improved Elman Neural Network for Daily Living Activities Recognition. 16th International Conference on Intelligent Systems Design and Applications, Dec 2016, Porto, Portugal. pp.697-707, 10.1007/978-3319-53480-0_69. hal-01462982

\section{HAL Id: hal-01462982 \\ https://hal.inria.fr/hal-01462982}

Submitted on 9 Feb 2017

HAL is a multi-disciplinary open access archive for the deposit and dissemination of scientific research documents, whether they are published or not. The documents may come from teaching and research institutions in France or abroad, or from public or private research centers.
L'archive ouverte pluridisciplinaire HAL, est destinée au dépôt et à la diffusion de documents scientifiques de niveau recherche, publiés ou non, émanant des établissements d'enseignement et de recherche français ou étrangers, des laboratoires publics ou privés. 


\title{
An Improved EIman Neural Network for Daily Living Activities Recognition
}

\author{
Zaineb Liouane $^{1,2}$, Tayeb Lemlouma ${ }^{2}$, Philippe Roose ${ }^{3}$, Fréderic Weis ${ }^{2}$ \\ And Hassani Messaoud ${ }^{1}$ \\ ${ }^{1}$ LARATSI, Monastir University, Ibn El Jazzar, Monastir, Tunisia, \\ ${ }^{2}$ IRISA, Rennes1 University, Rennes/Lannion, France, \\ ${ }^{3}$ LIUPPA/T2I, Pau and the adour countries University, Anglet, France,
}

\begin{abstract}
One of the main issues regarding the monitoring of persons in a smart home environment is the accuracy of the daily control of the person, the health prevention and the timely prediction of abnormal situations. To tackle this problem, this work proposes the use of an improved version of the Elman Neural Network (Elman-NN). In order to minimize the error between inputs and desired outputs, we optimize some criteria of the network to gain good results. We propose to use the Differential Evolution algorithm in the learning step of the Elman-NN to evolve the error performance. Our proposed model is responsible to predict the activities of the monitored elderly and to detect any state changes. This hybridization will help to optimize the weight and the bias of the network to achieve our objective function and to obtain a good network. The experimental results reveal that the proposed model is satisfactory for elderly person's movement prediction.
\end{abstract}

Keywords: Elman Neural Network; Differential Evolution algorithm; Prediction model; Smart home; Elderly person.

\section{Introduction}

The number of the elderly increased considerably each year. Aged people represent a growing part of the world population [1]. Since 2012, the number of older had increased to almost 810 million [2]. This situation motivates many research studies to focus a considerable interest on this aging population in order to provide required help and assistance. Smart home environnements represent an interesting and challenging way where such needed services are provided automatically (or semi automaticalltlly) by the smart system of the home. In a smart home, the occupant is continiouslly monitored using a set of sensors and ctuators. The sensors monitor the activities of the space's occupant and timely inform the system about the services needed depending to the current situation of the person and his health state. In order to ensure an efficient monitoring that considers the current context of an elderly and predict possible changes, the system must provide a better recognition of the humain activities, scenarios, health state and possible emergency situations. Several models and methods have been used to study the data issued from various sensors existing in a smart home. For instance, Support Vector Machines (SVMs), Markov Models [3], 
Artificial Neural Networks, Deep Neural Networks, Bayesian Network, etc. Unfortunately, the conventional previous methods did not succeed to solve the problem of the accuracy and the rate of certainty remains limited.

The objective of this work is to overcome the problem of the prediction accuracy. We focus on three features: the identification of the adequate recognition model to be used by selecting the appropriate artificial Neural Network, the architecture of the network and the learning algorithm. In order to build a new improved model with an efficient rate of prediction, we investigate the improvement of the Elman Neural Network (Elman-NN) [4] by using a learning algorithm belongs to the evolutionary algorithms there is the differential evolution (DE) algorithm. In order to accurately predict the monitored person's activities, actions and scenarios, we propose a prediction model based on Elman-NN and DE with multiple inputs. The advantages of the DE algorithm are its simple structure, ease of use, speed and robustness. DE is one of the best genetic algorithms for solving the issues of application which deal with real valued variables. The Elman-NN, based on the DE algorithm is used during the learning step and for the prediction of activities daily living (ADL).

The remainder of the paper is organized as follows: in section 2, a background of the prediction model is presented. In section 3, we describe the Elman-NN and the DE algorithm and present our proposed model. Section 4 presents the data source used in this work. The effectiveness of the Elman-NN based on the DE is discussed through simulations in section 5. Section 6 concludes this work.

\section{Related Works}

A large amount of the existing literature focuses on recognition models to monitor the activity of daily living of persons evolving in a smart home environment. Much research studied artificial networks in order to solve the problems of prediction wich is important in such environment to predict abnormal and risky situations.

Callaghan et al. [5], used the Echo State Network (ESN) to predict future data of time series in an intelligent space. Their results show that the training step of ESN is much faster than other kind of Recurrent Neural Networks (RNN). Fang et al. [6], proposed to use a Back Propagation Neural Network (BPNN) to recognize the activities of daily living in smart home. They used a feed forward strategy in the learning step and showed the impact of the number of used neurons in the error rate of accuracy. In the learning step of the Stable Neural Network (SNN), Teich et al. [7] proposed to use a feed forward neural network (Feed FNN) algorithm. The Feed FNN algorithm with a multiple hidden layers showed promising results. Oniga et al. [8], [9], used an ANN to predict the body posture of the person. In [9], the authors used two layer feed-forward network and a back-propagation algorithm in the learning step. Oniga et al. [9] concluded that for the hidden layer, 10 neurons and 5 neurons in the output layer give a good recognition rate. Xu et al. [10], proposed the application of particle swarm optimization algorithm to improve the Elman neural network in order to predict the wind power. The authors concluded that the proposed improved Elman-NN can overcome the problem of the local optimum and achieve a good prediction result with a high accuracy. 
Chong et al. [11], proposed to use a new model based on Elman-NN to improve the temperature drift modeling precision of a tuning fork micro-electromechanical system (MEMS) gyroscope. The results show that the multiple input/single output for Elman-NN based on GA can guarantee a high accurate modeling.

Previous related works show that the use of several types of the ANN promises good results in different domains, but most of the previous search use a deterministic algorithm in the learning step of the ANN such as a feed-forward algorithm, a backpropagation algorithm etc. Unfortunately several search shows that there are many inconvenient in training neural network with learning deterministic algorithms (gradient type) such as: The evaluation criterion is a differentiable function. In fact, when optimizing this criterion, a change of parameters is established in the direction of the gradient evaluation criteria from this parameter. The existences of a large number of sub-optimal solutions correspond to the local optima in the network. Deterministic algorithms are unable to give up a successful global optimum and do not confirm the completeness of network.

To overcome the previous limitation we propose to use the differential evolution algorithm in the learning step, it is non deterministic algorithm and it is autonomous (not require human intervention for the learning step). It can overcome the problem of local optima and leads to a global optimum with a high probability (thanks to their stochastic) and they have the property of completeness also we investigate the improvement of a specific type of the ANN that is the Elman Neural Networks for ADL recongnition .

\section{The Proposed Model}

\subsection{Elman Neural Network (EIman-NN)}

The Elman Neural Networks (Elman-NN) was proposed by Elman in 1990 [4]. Elman-NN is a dynamic recurrent network and is characterized by a multiple feed forward topology. Its architecture includes the input layer, a particular context node input layer, the hidden layer and the output layer. The main advantage of Elman-NN is that the context nodes can be used to memorize the previous data of the hidden nodes, which makes Elman-NN applicable in the fields of dynamic system identification and prediction control [12]. The structure of the Elman-NN can be presented in figure 1.

The network is characterized by a triplet $(W, B, f)$. Where $W$ is the weight, $B$ is the bias, and $f$ is the transfert function. Each node in these layers is linked using a the weighted connection $(W)$. Other parameters of Elman-NN are a Bias $(B)$ providing additional adjustable parameters of the model and the transfer function $(f)$ that calculates the output $(y)$. The transfer function can have different forms (Log function, Tan hyperbolic function, Linear function, Radial basis function, sigmoid function, etc.). 


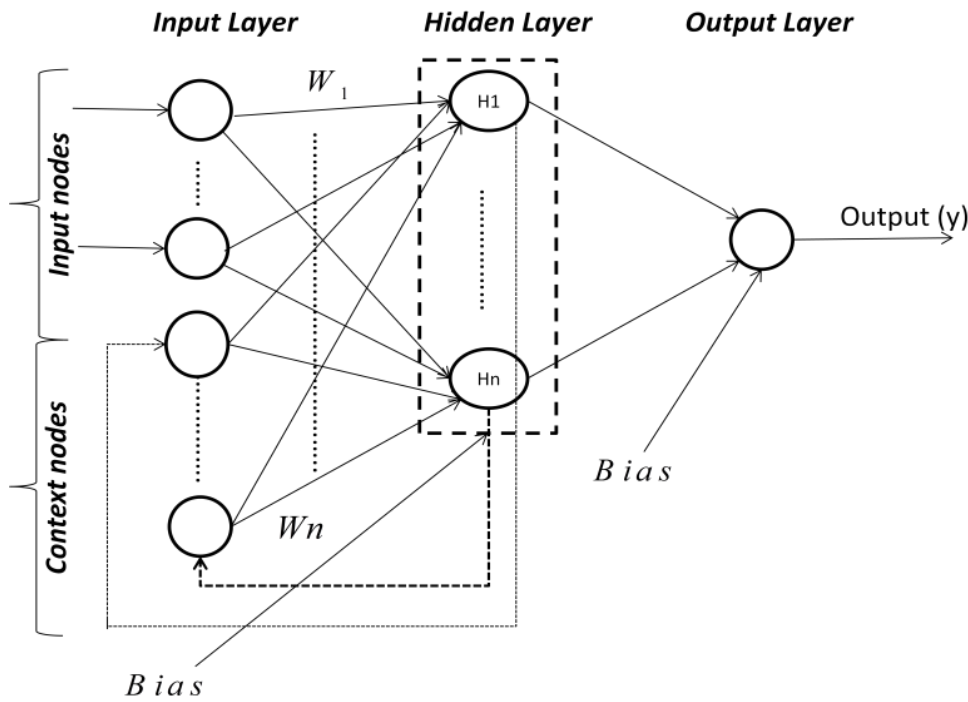

Fig. 1. Structure of Elman-Neural Network.

The Elman-NN is characterized by three steps: the initialization, the learning, and the validation step. The initialization step affects the accuracy and the rapidity of the prediction since the number of the hidden layers and the number of the hidden nodes enable to increase the certainty of the model, but in the other hand, increases the computation time. Consequently, the identification of the best structure of the network must be considered carefully. In addition to the impact of the learning step on the accuracy of the model; this step is responsible to adjust the parameter of the network (best weight and best bias). For this reason, in our work, we focus on the creation of a better structure of the network and the use an efficient learning algorithm. We propose to investigate the use of the Differential Evolution (DE) algorithm in the learning step of Elman-NN in order to increase the certainty of the model.

\subsection{Differential Evolution (DE) Algorithm}

The differential evolution (DE) algorithm proposed by Storn and Price [13], is a stochastic method inspired from the evolutionary algorithms. DE operates on a population of potential solutions by applying the principle of survival of the fittest to produce better approximations to a solution [14].

Firstly the DE algorithm produce a first/initial generation. If the optimization criterion is not satisfied, the creation of a new generation is applied. Individuals are selected according to their fitness for the production of new candidates (the offspring). Two or more candidates (called parents) are recombined to produce offspring. All offspring are transferred with a certain probability. The fitness of the offspring is then computed. The offspring are inserted into the population replacing the parents and producing a new generation. This cycle is then performed until the optimization criterion is reached. Such a single population evolutionary algorithm is 
powerful and performs well on a wide variety of problems. The used pseudo code of the $\mathrm{DE}$ algorithm is presented as follows.

\section{Pseudo-code of DE:}

Step I: generate an initial population containing $N P$ individuals each of them with a dimension $D$;

Step II: for each individual, choose a target vector $y(i)$ with a dimension $D$ from the Population;

Step III: randomly generate 3 vectors $\left(r_{1}, r_{2}, r_{3}\right)$ from the population different to $y(i)$;

Step IV: apply the mutation by finding the noisy vector $x(i)$. Many methods can be used to calculate the noisy vector. Here, we choose the following combination that is a basic mutation method: $x(i)=r_{1}+\mathrm{F}\left(r_{2}-r_{3}\right)$;

Step V: perform the crossover between $y(i)$ and $x(i)$ in order to find the trial vector $v(i)$

1. we choose a random number $\operatorname{rand}_{\mathrm{j}} \in[0,1]$ which is the $j^{\text {th }}$ value in the vector (chosen randomly among the population $\{1,2, . ., \mathrm{NP}\}$ ) and a CR which is the coefficient of the crossover which belongs to $[0,1]$ and determined by the user or the application;

2. If rand $_{\mathrm{j}}<\mathrm{CR}$ then $v(i) \leftarrow x(i)$ else $v(i) \leftarrow y(i)$;

Step VI: we calculate the costs of the trial $v(i)$ and the target vector $y(i)$;

Step VII: Once the cost is calculated, the vector that includes the lower cost replaces the population member in the initial population in case of minimization. Conversely, if the problem is maximization.

The previous code is the basic code of the DE algorithm which serves to evaluate the objective function, so in our work the objective function is to minimize the error between the input and the desired output. In the following part we discuss the use of the Elman-NN based on DE during the learning step.

\subsection{Elman-Neural Network based on DE}

In this work, we improve the Elman-NN using the DE algorithm for the learning step in order to tune the parameters of Elman-NN to improve the prediction accuracy. Our resulting model combines the Elman-NN and the DE algorithm and this hybridization focuses on the improvement of the Elman-NN and to boost the certainty of the prediction. Different criteria should be optimized in Elman-NN to obtain a good result by the $\mathrm{DE}$ algorithm in the learning step. Such criteria concern providing a better weight/bias of the network and minimizing the error of prediction. The flow chart of Elman-NN learning step based on DE algorithm in the learning step is shown in Figure 2. 


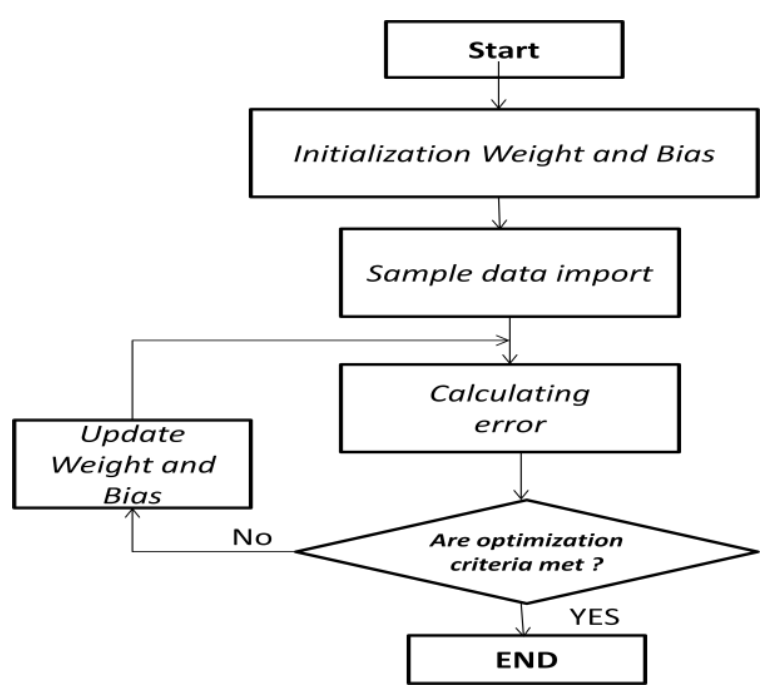

Fig.2. The flow chart of Elman-NN based on DE algorithm

This applied combination is responsible to optimize the weight and bias values in order to satisfy our objective function and obtain a minimum error in the prediction. The following pseudo-code (combination between the DE and Elman-NN) presents the use of the DE algorithm in the learning phase of Elman-NN.

Pseudo-code of Elman-NN based on DE:

Step I: a new population of individuals is randomly created;

Step II: we choose the target vector randomly $W_{\text {best }}$ and $B_{\text {best }}\left(W_{\text {best }}\right.$ and $B_{\text {best }}$ are considered as the best individuals);

Step III: randomly generate $W_{1}, W_{2}$ and $B_{1}, B_{2}$;

Step IV: create the noisy vector $W_{\mathrm{i}}$ and $B_{\mathrm{i}}$ for each individual in the population as follows: $W_{\mathrm{i}}=W_{\text {best }}+F\left(W_{1}-W_{2}\right)$ and $B_{\mathrm{i}}=B_{\text {best }}+F\left(B_{1}-B_{2}\right)$;

Step V: find the trial vector using a crossover between $W_{\text {best }}$ and $W_{\mathrm{i}}$, crossover between $B_{\text {best }}$ and $B_{\mathrm{i}}$;

Step VI: calculates the cost of the target vector and the trial vector by Elman-NN;

Step VII: the vector that includes the lower cost replaces the population member in the initial population. Repeat the different steps until funding the best cost of prediction (with a minimum error);

Thanks to this learning step, we are able to identify the best model having the best parameters (weight and bias) of the networks which implicitly influence on our objective function that is the minimization of the error rate between the predicted and desired data. Indeed, the model obtained after the learning step provides us a minimum error of prediction. After the learning step, our model is ready to achive the prediction used during the monitoring in a smart home environment. In our framework, we apply the prediction on the movements of monitored person with minimizing the cost of errors. 


\section{Data Source}

In order to test efficiently our proposed model, i.e. the accuracy and the error of prediction, we propose to use a dataset collected by different sensors existing in different spaces of a smart home. The objective here is to predict the activities of daily living, the health perturbation and the abnormal activities of a monitored elderly person. In this work, the dataset used comes from a health monitoring project "ehealth monitoring open data project" where data represents scenarios of an elderly person in a smart home monitored during one year. Each input data includes three parameters: starting time - ending time - code of activities and actions. Table 1 shows the different activities listed by their code. From Table 1, we can note that for the grooming and meal preparation activities there are different codes in the same kind of activity, each code presents a detailed action.

For grooming activity \{Washing hand/face (4), Hair dray (5), Move dish (34), Make up (6)\}.

For Meal preparation activity \{Wash dish (11), Make coffee (13), Make tee (14), Make sandwich (15), Make hot food (16)\}.

Table 1. Activities of the monitored person classed by code

\begin{tabular}{|l|l|}
\hline Codes & Activities \\
\hline 1 & Eating \\
\hline 2 & Dressing \\
\hline 3 & washing \\
\hline$\{6-4-5-34\}$ & grooming \\
\hline 7 & Unary-function \\
\hline 8 & Bowel-function \\
\hline 9 & Toileting \\
\hline 10 & House-keeping \\
\hline 11 & Landry \\
\hline$\{13-14-15-16\}$ & Meal preparation \\
\hline 17 & Telephone \\
\hline 18 & Medication Use \\
\hline 19 & Watching TV \\
\hline 24 & Sleeping \\
\hline 33 & Go out \\
\hline 35 & Reading \\
\hline
\end{tabular}

It is rather difficult to directly identify and extract the movement information of the monitored person from the collected sensor data existing in the input dataset seeing that a complex and heterogeneous data. For this reason; we firstly convert the input data in the form of time series indicating the different activities of the elderly at home. A time serie is a sequence of data, typically consisting of successive measurements made over a time interval. Secondly, we normalize the input data between 0 and 1 to be ready as inputs for the predictive techniques. 
The value of the input data is normalized as:

$$
\bar{X}=\frac{X-\min (\mathrm{X})}{\max (\mathrm{X})-\min (\mathrm{X})}
$$

Where $\mathrm{X}$ is the current values, $\max (\mathrm{X})$ is the largest value and $\min (\mathrm{X})$ is the smallest value of the sample.

\section{Simulation and Results}

To efficiently create a good network of the Elman-NN based on DE we choose firstly the structure of the model (input, output and hidden neurons number). We analyze the results of the Elman-NN-DE using 8 input neurons, 9 output neurons and 8 hidden neurons during 30 days. In order to evaluate the performance of the ElmanNN-DE we calculate the prediction error. For this, we evaluate the root mean square error (RMSE) given by:

$$
R M S E=\sqrt{\frac{1}{N} \sum_{i=1}^{N}\left(Y_{i}-X_{i}\right)^{2}}
$$

Where $N$ represents the number of the learning observations, $Y_{\mathrm{i}}$ represents the output network, and $X_{\mathrm{i}}$ represents the input value for the i observation (with $i$ from 1 to $N$ ). After the simulation of the Elman-NN based on DE, the observerd result of the RMSE is $2.5710^{*} 10^{-5}$ which is a good result that shows the certainty of our model.

To confirm the performance of our proposed model in the prediction of the elderly activities in the smart home, we compare our Elman-NN based on DE algorithm model with Elman-NN based on back-propagation algorithm and Elman-NN based on Genetic algorithm. Table2 shows that our model succedes to provide a better result if compared with the other algorithms during the learning step. Indeed, the results show that the RMSE of the prediction using Elman-NN based on Genetic Algorithm is 0.0145 while for Elman-NN based on multi-layer back-propagation algorithm the RMSE is 2.7800. Our proposed model Elman-NN based on DE provides a RMSE of $2.5710 * 10^{-5}$ which improve significantly the prediction.

Table 2. Comparison of the RMSE between Elman-NN based on GA, Elman-NN based on multi-layer-back-propagation algorithm and Elman-NN based on DE.

\begin{tabular}{|c|c|c|c|}
\hline $\begin{array}{c}\text { Prediction } \\
\text { Models }\end{array}$ & $\begin{array}{c}\text { Elman-NN based } \\
\text { on GA }\end{array}$ & $\begin{array}{c}\text { Elman-NN based on multi- } \\
\text { layer back-propagation } \\
\text { algorithm }\end{array}$ & $\begin{array}{c}\text { Elman-NN } \\
\text { based on DE }\end{array}$ \\
\hline RMSE & 0.0145 & 2.7800 & $2.5710^{*} 10^{-5}$ \\
\hline
\end{tabular}


As observed, the use of the differential evolution algorithm in the learning step decreases the root mean square error, i.e. increases the certainty of the prediction. To study the performance of our proposed model, we compared the cost of error during the learning step between Elman-NN based on GA and Elman-NN based on DE, since $\mathrm{DE}$ and GA belong to the same algorithm class of evolutionary/ stochastic algorithms (Figure 3).

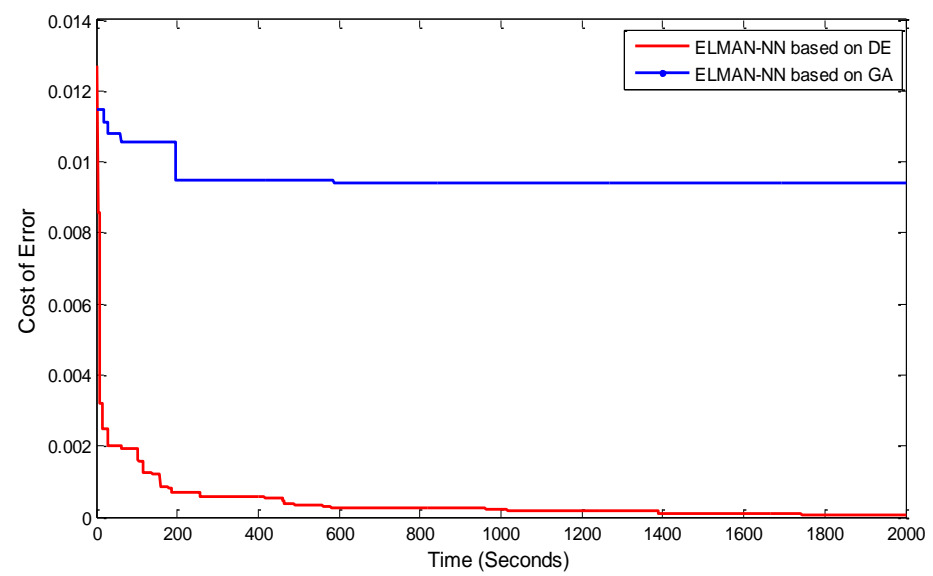

Fig.3. Comparison of the cost of error between Elman-NN based on GA and Elman-NN based on DE.

From Figure 3, we observe that the cost of error of the Elman-NN based on DE is very small compared to the cost of error of Elman-NN based on GA. Moreover, we can see that the error rate with DE starts to decrease rapidly against the error rate with GA which decreased slowly.

These results confirm the performance of Elman-NN based on DE in term of accuracy and rapidity during the training step. Regarding the validation step of our porposed model, we propose to test the accuracy of our prediction data separately in defferent times of the day: the morning, evening, afternoon and night. Figure 4 presenst four curves where each one shows the the real data and predicted data during different periods of the day . 


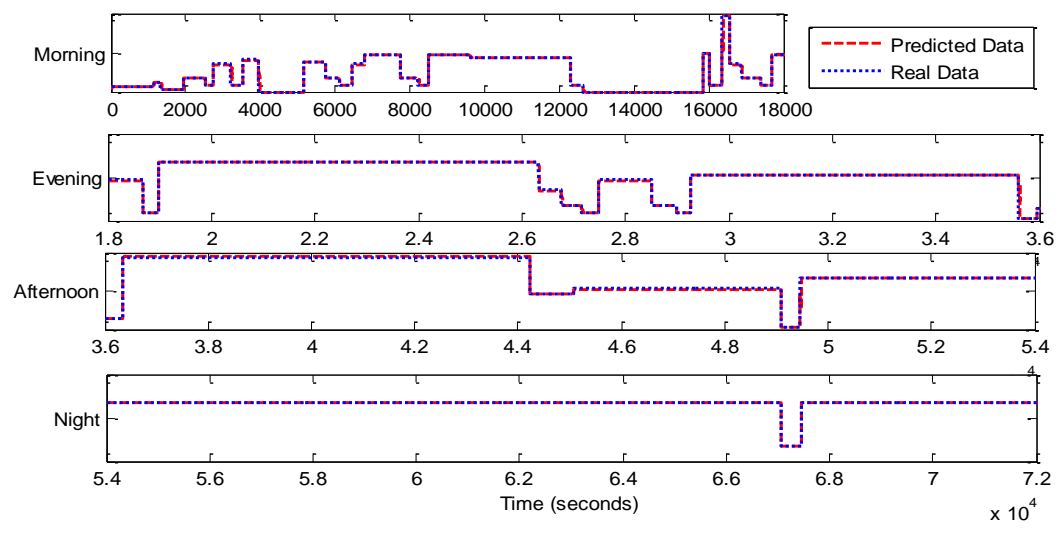

Fig.4. The predicted and the real data in four times of the day (Morning, Evening, Afternoon, Night).

The results of the validation step show that the shape of the predicted curve is very similar to the real data curve. This result confirms the accuracy of Elman-NN based on DE at different times of the day. Consequentlly, our improved model succeded to perform a continuous monitoring with a high accuracy.

\section{Conclusion}

In this paper, we proposed a hybrid model based on Elman Neural Network and differential evolution (DE) algorithm in order to predict efficiently and with great accuracy the activities of daily living of a monitored person.

Elman-NNs have been widely used in various areas, but the accuracy of results remained an opened issue. In order to overcome these problems, we focused this work on the optimization the the Elman-NN during learning step; we investigated the use the DE algorithm in the learning step of the Elman-NN to improve the accuracy and the efficiency of the prediction activities.

The results of our simulations show that the Elman-NN based on the DE model provided a better accuracy, higher efficiency and a significant rapidity if compared to the Elman-NN based on a back-propagation algorithm or the Elman-NN based on genetic algorithm. The obtained results of using Elman-NN based on DE are promising. 
Acknowledgments. The authors would like to thank all teams of the project " e-health monitoring open data project".

\section{References}

1. Undesa. : Population Division. In : United Nations Department of Economic and Social Affairs, 2012.

2. Skouby, K. E., Kivimäki, A., Haukiputo, Lotta., et al.: Smart Cities and the Ageing Population. In : The 32nd Meeting of WWRF. 2014.

3. Zaineb, L., Tayeb, L., Philippe, R., Fréderic, W., and Hassani, M.: A Markovianbased Approach for Daily Living Activities Recognition. In: International Conference on Sensor Networks (SENSORNETS), 2016

4. Elman, J. L.: Finding structure in time. Cognitive science, 1990, vol. 14, no 2, p. 179211.

5. Callaghan, V., et al.: Echo State Network for Occupancy Prediction and Pattern Mining in Intelligent Environments. In : 5th International Conference on Intelligent Environments, Barcelona 2009. IOS Press, 2009. p. 474.

6. Fang, H., He, L.: BP neural network for human activity recognition in smart home. In : 2012 International Conference on Computer Science and Service System. 2012.

7. Liu, Z., Song, Y., Shang, Y. : Posture recognition algorithm for the elderly based on BP neural networks. In : The 27th Chinese Control and Decision Conference (2015 CCDC). IEEE, 2015. p. 1446-1449.

8. Oniga, S., Sütö, J. : Human activity recognition using neural networks. In : 15 th International Carpathian Control Conference (ICCC) IEEE, 2014. p. 403-406.

9. Oniga, S., Suto, J.: Activity Recognition in Adaptive Assistive Systems Using Artificial Neural Networks. In : Elektronika ir Elektrotechnika, 2016, vol. 22, no 1, p. 68-72.

10. Xu, L., Mao, J.: Short-term wind power forecasting based on Elman neural network with particle swarm optimization. In : 2016 Chinese Control and Decision Conference (CCDC). IEEE, 2016. p. 2678-2681.

11. Chong, S., Rui, S., Jie, L.: Temperature drift modeling of MEMS gyroscope based on Genetic-Elman neural network. In : Mechanical Systems and Signal Processing, 2016, vol. 72, p. 897-905.

12. Yongchun, L.: Application of Elman neural network in short-term load forecasting. In : Artificial Intelligence and Computational Intelligence (AICI), 2010 International Conference on. IEEE, 2010. p. 141-144.

13. Storn, R., Price, K.: Differential evolution-a simple and efficient heuristic for global optimization over continuous spaces. In : Journal of global optimization, 1997, vol. 11, no 4, p. 341-359. 